\title{
Does Stem Cell Implantation Have an Effect on Severity of Retinitis Pigmentosa: Evaluation with a Classification System?
}

\author{
Ayşe Öner, Neslihan Sinim Kahraman \\ Ophthalmology Department, Acibadem Kayseri Hospital, Kayseri, Turkey \\ Email: ayseozoner@gmail.com
}

How to cite this paper: Öner, A. and Kahraman, N.S. (2021) Does Stem Cell Implantation Have an Effect on Severity of Retinitis Pigmentosa: Evaluation with a Classification System? Open Journal of Ophthalmology, 11, 36-48.

https://doi.org/10.4236/ojoph.2021.111004

Received: December 23, 2020

Accepted: February 22, 2021

Published: February 25, 2021

Copyright $\odot 2021$ by author(s) and Scientific Research Publishing Inc. This work is licensed under the Creative Commons Attribution International License (CC BY 4.0).

http://creativecommons.org/licenses/by/4.0/

\begin{abstract}
Background: Cell replacement therapies have been evaluated in recent years as an alternative for various retinal pathologies to evaluate the therapeutic efficacy of cell therapy, it is important to measure the severity of the disease. The aim of this study was to evaluate the effect of umbilical cord derived Mesenchymal Stem Cell (UC-MSC) implantation on severity of Retinitis Pigmentosa (RP). Methods: This single-center, clinical study included data of 138 eyes of 92 patients who had a confirmed diagnosis of RP and received stem cell implantation to the suprachoroidal area with a surgical procedure. Patients were evaluated before and 1 year after the surgery regarding to the outcome measures of Best Corrected Visual Acuity (BCVA), Optical Coherence Tomography (OCT) and Visual Field (VF) tests. BCVA, VF width and ellipsoid zone (EZ) width on OCT were recorded for each patient and a scoring criterion was established for each variable varying from 0 to 5 depending on its distribution. The cumulative score (from 0 to 15) was used to classify disease severity from grade 0 to 5 . Results: All of the patients completed 12-month follow-up period. The median age of the patients was 40.8 years, $46 \%$ were female, $77 \%$ had been diagnosed within 10 years and $41 \%$ had a family history. $79 \%$ of the patients with family history had autosomal recessive inheritance pattern. There were statistically significant improvements in the mean BCVA and VF scores during the study $(\mathrm{p}<0.05)$. The mean score and the mean grade of the disease also improved after the treatment $(\mathrm{p}<$ 0.05). There was a negative correlation between BCVA improvement and scoring and grading of the disease. Conclusions: This study demonstrated beneficial effect of suprachoroidally applied UC-MSCs on BCVA, VF and the severity score and grade of the disease during 12-month follow-up period. Cell mediated therapy based on the secretion of Growth Factors (GFs) seems to be an effective and safe option for the treatment of degenerative retinal
\end{abstract}


diseases. This classification is simple, produces objective measure of disease severity and gives opportunity to compare the results of different treatment modalities.

\section{Keywords}

Cell Mediated Therapy, Retinitis Pigmentosa, Suprachoroidal Implantation, Umbilical Cord Derived Mesenchymal Stem Cell, Visual Function, Classification

\section{Introduction}

Retinitis Pigmentosa (RP) is a complex group of hereditary retinal disorders, which causes degeneration of retinal photoreceptors. It has been reported that the disease has a worldwide prevalence of $1 / 4000$ and it can be inherited with different patterns. The disease initially begins with night blindness and progresses to loss of vision. Up to now, there is no definitive curative treatment for patients with RP [1] [2].

Cell replacement therapies have been evaluated in recent years as an alternative for various retinal pathologies [3] [4]. Mesenchymal Stem Cells (MSCs) are undifferentiated cells which have the ability to provide trophic support for neuroprotection and regeneration of damaged retinal cells either directly through the secretion of Neurotrophic Factors (NTFs) or indirectly with the paracrine support [3] [4]. Recent clinical and experimental studies reported that umbilical cord-derived MSCs (UC-MSCs) are effective in preventing retinal degeneration and rescuing photoreceptors [5] [6].

To evaluate the therapeutic efficacy of cell therapy, it is important to measure the severity of the disease. As well as the clinical assessment and visual acuity measurement, the disease can be examined by a variety of tests like Optical Coherence Tomography (OCT), Visual Field (VF) and Electroretinography (ERG) [7] [8]. Based on the data obtained from previous clinical studies, we conducted a single-center, clinical study in patients with RP to investigate the effect of suprachoroidally implanted UC-MSCs to the severity of the disease.

In a recent study, Iftikhar et al. [9] designed a classification based on the findings obtained from basic clinical examination and available diagnostic tests. This classification is simple, easy, widely applicable to all patients and provides objective data about the severity of the disease. It also gives opportunity to compare the results of different treatment modalities. The aim of this study was to evaluate the effect of UC-MSC implantation to the severity of RP by using this classification system.

\section{Methods}

\subsection{Study Design and Setting}

This study was conducted to investigate the effect of suprachoroidal UC-MSC 
implantation to the severity of RP by using a phenotypic classification system. The study was performed in the ophthalmology department of a tertiary hospital between 01.03.2019 and 06.30.2020 in accordance with the Declaration of Helsinki, after obtaining the approval of the Ethics Committee of the University $(2017 / 480,10.13 .2017)$ and the approval by the Review Board of Stem Cell Applications of the Ministry of Health (Registration number: 56733164/203) according to the regulations in our country. Written informed consent was obtained from the participants of the study.

\subsection{Patients}

After receiving a complete medical history including patient demographics, disease duration, inheritance pattern, the patients received a detailed ophthalmic examination including BCVA, intraocular pressure measurement, anterior segment evaluation with slit-lamp biomicroscopy, color fundus photography, OCT and VF. BCVA was recorded with a Snellen chart at a distance of 3 meters. VF examination was performed by Humphrey VF analyzer device (Carl Zeiss Meditec AG Germany), program 30-2 was used for testing of each eye.

\subsection{The Stem Cell Preparation and Surgical Procedure}

Stem Cell Preparation: The production protocol of UC-MSCs was previously described elsewhere [10]. Umbilical cord was disinfected, cut into pieces of 1 - 2 $\mathrm{mm}^{2}$ and transferred to $75 \mathrm{~cm}^{2}$ culture flasks in Dulbecco's modified Eagle's medium-low glucose (DMEM-LG) containing a concentration of $10 \%$ human serum and of $1 \%$ penicillin/streptomycin. The cells were cultured at $37^{\circ} \mathrm{C}$ with $5 \%$ density of $\mathrm{CO}_{2}$. Every 3 day, the culture medium was removed and fresh medium was added until the cells reached minimum $70 \%$ confluency. After the third passage, the cells were analyzed with flow cytometry for confirmation of phenotypic characteristics. The UC-MSCs were positive for CD-73, CD-90, and CD-105, and negative for CD-34, CD-45, and HLA-DR. The preparation procedure was performed under good manufacturing practice (GMP) conditions and before release UC-MSCs were assessed for cell appearance, viability, identification, purity, content, and potency. The cells were also screened for bacterial or fungal contamination. Cell viability was $>90.0 \% \pm 0.5 \%$ before cell transplantation. A concentration of $5 \times 10^{6}$ cells in isotonic solution containing $1 \%$ human serum albumin were carried in $2 \mathrm{~mL}$ vials which was placed in a sterile tube. The tubes were transfered with the temperature-controlled bag (set at a temperature between $2^{\circ} \mathrm{C}-8^{\circ} \mathrm{C}$ ) until the cells reach the operating room. The cells were used in $24 \mathrm{~h}$.

We performed a surgical technique defined as the Limoli retinal restoration technique (LRRT), described by Limoli et al. [11] [12]. An experienced surgeon (A.O) performed all surgeries. All operations were done with local anesthesia. The details of the surgery were as follows: The globe was deviated to the supero-nasal quadrant and conjunctiva was dissected at the infero-temporal quadrant at $8 \mathrm{~mm}$ from the limbus. A deep scleral flap of about $5 \times 5 \mathrm{~mm}$ was 
opened by radial hinge at the infero-temporal quadrant. The sclerectomy was deep enough to allow viewing of the color of the choroid. A flap from the orbital fat was extracted from a gap above the inferior oblique muscle. This tissue was laid on the scleral bed and sutured with $6 / 0$ vicryl at the proximal edge. The scleral flap was then sutured above the fat pedicle. The remaining space between the autologous fat graft, choroid, and scleral flap was filled with $1 \mathrm{cc}$ of $5 \times 10^{6}$ UC-MSCs using a 25 -gauge cannula. The conjunctiva was sutured with $8 / 0$ vicryl.

Patients were recommended to use topical antibiotic and steroid eye drops four times a day for 1 month after surgery. Routine ophthalmic evaluations and OCT were performed before surgery, and at the postoperative 1st day, 1st week, 1st, 3rd, 6th and 12th months. We also performed VF and color fundus photographs at 1st, 3rd, 6th and 12th months, FFA and mfERG at 6th and 12th months postoperatively. All patients were evaluated for adverse events of the surgical procedures during the study period.

\subsection{Severity Classification}

Patients were evaluated before and 1 year after the surgery regarding to the outcome measures of BCVA, OCT and VF tests. BCVA, VF width, ellipsoid zone (EZ) width on OCT were recorded for each patient and a scoring criterion was established for each variable varying from 0 to 5 depending on its distribution. The cumulative score (from 0 to 15) was used to classify disease severity from grade 0 to 5 (Table 1 and Table 2). We also evaluated the Mean Deviation (MD)

Table 1. Scoring criteria.

\begin{tabular}{cccc}
\hline Score & $\begin{array}{c}\text { Visual Acuity } \\
\text { (ETDRS letter score/Snellen) }\end{array}$ & $\begin{array}{c}\text { Visual Field Diameter } \\
\left({ }^{\circ}\right)\end{array}$ & $\begin{array}{c}\text { Elipsoid Zone Width } \\
\left({ }^{\circ}\right)\end{array}$ \\
\hline 0 & $\geq 85(20 / 20)$ & $\geq 120$ & $<30$ \\
1 & $<85(20 / 20)$ to $\geq 80(20 / 25)$ & $<120$ to $\geq 80$ & $<15$ to $\geq 10$ \\
2 & $<80(20 / 25)$ to $\geq 75(20 / 32)$ & $<80$ to $\geq 40$ & $<10$ to $\geq 7$ \\
3 & $<75(20 / 32)$ to $\geq 70(20 / 40)$ & $<40$ to $\geq 20$ & $<7$ to $\geq 5$ \\
4 & $<70(20 / 40)$ to $\geq 55(20 / 100)$ & $<20$ to $\geq 15$ & $<5$ \\
5 & $<55(20 / 100)$ & $<15$ & $<$ \\
\hline
\end{tabular}

Table 2. Grading criteria.

\begin{tabular}{cc}
\hline Grade & Cumulative score \\
\hline 0 & 0 \\
1 & $1-3$ \\
2 & $4-6$ \\
3 & $7-9$ \\
4 & $10-12$ \\
5 & $13-15$ \\
\hline
\end{tabular}


value of VF and Central Macular Thickness (CMT) measurements on OCT for each patient.

Severity classification was done according to the system designed by Iftikhar et al. [9]. BCVA was given a score of 0 if vision was $\geq 20 / 20 ; 1$ if it was $<20 / 20$ to $\geq 20 / 25 ; 2$ if it was $<20 / 25$ to $\geq 20 / 32$; 3 if it was $<20 / 32$ to $\geq 20 / 40 ; 4$ if it was $<20 / 40$ to $\geq 20 / 100$; and 5 if it was $<20 / 100$. VF diameter was given a score of 0 if it was $\geq 120^{\circ}$ or better; 1 if it was $<120^{\circ}$ to $\geq 80^{\circ} ; 2$ if it was $<80^{\circ}$ to $\geq 40^{\circ} ; 3$ if it was $<40^{\circ}$ to $\geq 20^{\circ} ; 4$ if it was $<20^{\circ}$ to $\geq 15^{\circ}$; and 5 if it was $<15^{\circ}$. EZ was given a score of 0 if it was $\geq 30^{\circ}$ (occupying the whole width of the OCT scan); 1 if it was $<30^{\circ}$ to $\geq 15^{\circ} ; 2$ if it was $<15^{\circ}$ to $\geq 10^{\circ} ; 3$ if it was $<10^{\circ}$ to $\geq 7^{\circ} ; 4$ if it was $<7^{\circ}$ to $\geq 5^{\circ}$; and 5 if it was $<5^{\circ}$. Table 1 shows the scoring criteria for each variable and Table 2 includes the grading scale. Data of 138 eyes of 92 patients who received stem cell therapy were evaluated according to the classification system.

\subsection{Statistical Analysis}

Statistical analyses were conducted using SPSS version 20 statistical package program (IBM Corp. in Armonk, NY). Descriptive data are presented as median with interquartile range for non-normally distributed numerical variables, and as the frequencies and percentage for categorical variables. Shapiro-Wilk and Kolmogorov-Smirnov tests were used to evaluate the distribution of the numeric data. Pearson's Chi-Square test and One-Way ANOVA test was used for comparing the numeric data with a Scheffe test for post-hoc pairwise comparison. $\mathrm{p}$ $<0.05$ was considered as statistically significant level.

\section{Results}

Data of 138 eyes of 92 patients who completed 12-month follow-up period were reviewed. The mean age was 40.8 years and $46 \%$ of the study group were female. The mean age at onset of disease was 19.4 years and the mean disease duration was 21.8 years. There was a family history in $41 \%$ of the patients. Among the patients with a family history autosomal dominant pattern was found in $21 \%$ and autosomal recessive pattern was found in $79 \%$ of the patients (Table 3). Demographic data of the patients was shown in Table 3. Genetic results for causative mutations were available for $14(15 \%)$ patients.

There were statistically significant improvements in BCVA, BCVA score, CMT, VF score, VF-MD value, score and grade of the disease after treatment ( $\mathrm{p}$ $<0.05)$. The EZ score did not show any significance $(\mathrm{p}>0.05)$ (Table 4).

OCT evaluations showed abnormalities in 5 patients before surgery. $3 \mathrm{had}$ Cystoid Macular Edema (CME), 1 had epiretinal membrane and 1 had Vitreomacular Traction (VMT). VMT and CME resolved in 2 patients after surgery. There were no morphological changes in OCT scans of other patients. The mean CMT measurements of the all treated eyes increased significantly after treatment $(\mathrm{p}<0.05)$.

Distributions of the grades before surgery were as follows: There were no eyes classified as grade 0 , and grade 1.3 eyes (2\%) were classified as grade 2, 2 eyes 
Table 3. Demographics of the patients.

\begin{tabular}{cc}
\hline Characteristics $(\mathbf{n}=\mathbf{9 2})$ & \\
\hline Age (years), mean \pm SD & $40.8 \pm 12.8$ \\
$\quad$ Sex (male), $\mathrm{n}(\%)$ & $50(54.0)$ \\
Age of onset (years) mean \pm SD & $19.4 \pm 8.9$ \\
Duration of the disease (years) mean \pm SD & $21.8 \pm 9.5$ \\
Family History (+/-) & $38 / 54$ \\
\hline Mode of Inheritance & $8(21)$ \\
Autosomal dominant & $30(79)$ \\
\hline
\end{tabular}

SD: Standart Deviation.

Table 4. Comparison of the preoperative and postoperative variables.

\begin{tabular}{|c|c|c|c|c|c|c|}
\hline \multirow[b]{2}{*}{ Variables } & \multirow[b]{2}{*}{$\mathrm{n}$} & \multicolumn{2}{|c|}{ Preoperative } & \multicolumn{2}{|c|}{ Postoperative } & \multirow[b]{2}{*}{$\mathrm{p}$} \\
\hline & & Mean \pm SD & $\begin{array}{c}\text { Median } \\
(\text { Min - Max })\end{array}$ & Mean \pm SD & $\begin{array}{c}\text { Median } \\
(\text { Min - Max })\end{array}$ & \\
\hline $\begin{array}{l}\text { Visual acuity (VA) } \\
\text { (Snellen Lines) }\end{array}$ & 138 & $0.11 \pm 0.17$ & $\begin{array}{c}0.05 \\
(0.00-0.80)\end{array}$ & $0.16 \pm 0.21$ & $\begin{array}{c}0.05 \\
(0.00-0.90)\end{array}$ & $<0.05$ \\
\hline VA Score & 138 & $4.7 \pm 0.7$ & $\begin{array}{c}5.0 \\
(2.0-5.0)\end{array}$ & $4.5 \pm 0.9$ & $\begin{array}{c}5.0 \\
(1.0-5.0)\end{array}$ & $<0.05$ \\
\hline $\mathrm{CMT}(\mu \mathrm{m})$ & 138 & $118.6 \pm 52.7$ & $\begin{array}{c}114.5 \\
(38.0-388.0)\end{array}$ & $123.0 \pm 50.6$ & $\begin{array}{c}118.0 \\
(40.0-383.0)\end{array}$ & $<0.05$ \\
\hline EZ Score & 138 & $4.8 \pm 0.6$ & $\begin{array}{c}5.0 \\
(0.0-5.0)\end{array}$ & $4.8 \pm 0.6$ & $\begin{array}{c}5.0 \\
(0.0-5.0)\end{array}$ & $>0.05$ \\
\hline VF Score & 138 & $4.5 \pm 1.0$ & $\begin{array}{c}5.0 \\
(1.0-5.0)\end{array}$ & $4.2 \pm 1.3$ & $\begin{array}{c}5.0 \\
(0.0-5.0)\end{array}$ & $<0.05$ \\
\hline VF-MD Value (dB) & 138 & $30.50 \pm 4.43$ & $\begin{array}{c}32.27 \\
(6.43-34.60)\end{array}$ & $29.94 \pm 4.84$ & $\begin{array}{c}31.73 \\
(6.27-34.65)\end{array}$ & $<0.05$ \\
\hline Score of the disease & 138 & $14.0 \pm 1.7$ & $\begin{array}{c}15.0 \\
(4.0-15.0)\end{array}$ & $13.4 \pm 2.0$ & $\begin{array}{c}14.0 \\
(4.0-15.0)\end{array}$ & $<0.05$ \\
\hline Grade of the disease & 138 & $4.8 \pm 0.5$ & $\begin{array}{c}5.0 \\
(2.0-5.0)\end{array}$ & $4.6 \pm 0.6$ & $\begin{array}{c}5.0 \\
(2.0-5.0)\end{array}$ & $<0.05$ \\
\hline
\end{tabular}

There were statistically significant improvements in BCVA, BCVA score, CMT, VF score, VF-MD value, score and grade of the disease after treatment $(\mathrm{p}<0.05)$. The EZ score did not show any significance $(\mathrm{p}>$ 0.05). VA: Visual acuity; CMT: Central macular thickness; $\mu \mathrm{m}$ : micrometer; EZ: Ellipsoid zone; VF: Visual field; VF-MD Value: Visual field mean deviation value; dB: decibel.

(1\%) as grade 3, 23 eyes (17\%) as grade 4 and 110 eyes (80\%) as grade 5. After stem cell treatment the distribution changed as follows: There were no eyes classified as grade 0,1 eye $(0.5 \%)$ as grade 1,3 eyes (2\%) as grade 2,6 eyes (4\%) as grade 3,45 eyes $(32.5 \%)$ as grade 4 and 83 eyes $(61 \%)$ as grade 5 . The improvements in grading of the disease after treatment were statistically significant $(\mathrm{p}<$ 0.05) (Table 5). 
Table 5. The distribution of scores of the patients before and after surgery.

\begin{tabular}{|c|c|c|c|c|c|c|c|c|c|c|c|c|c|}
\hline \multirow{2}{*}{ Variables } & \multicolumn{6}{|c|}{ Preoperative } & \multicolumn{6}{|c|}{ Postoperative } & \multirow{2}{*}{$\mathrm{p}$} \\
\hline & 0 & 1 & 2 & 3 & 4 & 5 & 0 & 1 & 2 & 3 & 4 & 5 & \\
\hline VA Score & $\begin{array}{c}0 \\
(0 \%)\end{array}$ & $\begin{array}{c}0 \\
(0 \%)\end{array}$ & $\begin{array}{c}5 \\
(4 \%)\end{array}$ & $\begin{array}{c}4 \\
(2 \%)\end{array}$ & $\begin{array}{c}22 \\
(16 \%)\end{array}$ & $\begin{array}{c}107 \\
(78 \%)\end{array}$ & $\begin{array}{c}0 \\
(0 \%)\end{array}$ & $\begin{array}{c}2 \\
(1 \%)\end{array}$ & $\begin{array}{c}6 \\
(4.5 \%)\end{array}$ & $\begin{array}{c}6 \\
(4.5 \%)\end{array}$ & $\begin{array}{c}34 \\
(25 \%)\end{array}$ & $\begin{array}{c}90 \\
(65 \%)\end{array}$ & $<0.05$ \\
\hline EZ Score & $\begin{array}{c}1 \\
(0.5 \%)\end{array}$ & $\begin{array}{c}0 \\
(0 \%)\end{array}$ & $\begin{array}{c}0 \\
(0 \%)\end{array}$ & $\begin{array}{c}4 \\
(3 \%)\end{array}$ & $\begin{array}{c}14 \\
(10 \%)\end{array}$ & $\begin{array}{c}119 \\
(86 \%)\end{array}$ & $\begin{array}{c}1 \\
(0.5 \%)\end{array}$ & $\begin{array}{c}0 \\
(0 \%)\end{array}$ & $\begin{array}{c}0 \\
(0 \%)\end{array}$ & $\begin{array}{c}4 \\
(2 \%)\end{array}$ & $\begin{array}{c}20 \\
(14.5 \%)\end{array}$ & $\begin{array}{c}114 \\
(83 \%)\end{array}$ & $>0.05$ \\
\hline VF Score & $\begin{array}{c}0 \\
(0 \%)\end{array}$ & $\begin{array}{c}1 \\
(0.5 \%)\end{array}$ & $\begin{array}{c}9 \\
(6.5 \%)\end{array}$ & $\begin{array}{c}9 \\
(6.5 \%)\end{array}$ & $\begin{array}{c}16 \\
(11.5 \%)\end{array}$ & $\begin{array}{c}103 \\
(75 \%)\end{array}$ & $\begin{array}{c}0 \\
(0 \%)\end{array}$ & $\begin{array}{c}9 \\
(6.5 \%)\end{array}$ & $\begin{array}{c}8 \\
(6 \%)\end{array}$ & $\begin{array}{c}16 \\
(11.5)\end{array}$ & $\begin{array}{c}19 \\
(14 \%)\end{array}$ & $\begin{array}{c}86 \\
(62 \%)\end{array}$ & $<0.05$ \\
\hline $\begin{array}{l}\text { Grade of } \\
\text { the disease }\end{array}$ & $\begin{array}{c}0 \\
(0 \%)\end{array}$ & $\begin{array}{c}0 \\
(0 \%)\end{array}$ & $\begin{array}{c}3 \\
(2 \%)\end{array}$ & $\begin{array}{c}2 \\
(1 \%)\end{array}$ & $\begin{array}{c}23 \\
(17 \%)\end{array}$ & $\begin{array}{c}110 \\
(80 \%)\end{array}$ & $\begin{array}{c}0 \\
(0 \%)\end{array}$ & $\begin{array}{c}1 \\
(0.5 \%)\end{array}$ & $\begin{array}{c}3 \\
(2 \%)\end{array}$ & $\begin{array}{c}6 \\
(4 \%)\end{array}$ & $\begin{array}{c}45 \\
(32.5 \%)\end{array}$ & $\begin{array}{c}83 \\
(61 \%)\end{array}$ & $<0.05$ \\
\hline
\end{tabular}

The improvements in grading of the disease after treatment were statistically significant $(\mathrm{p}<0.05)$. VA: Visual acuity; EZ: Elipsoid zone; VF: Visual field.

\section{Discussion}

$\mathrm{RP}$ is one of the leading causes of severe visual impairment in young individuals. Low vision is known to complicate daily life, and chronic visual deterioration caused by degenerative retinal diseases have been shown to affect quality of life [13].

Examining the retinal structure and visual function of patients allows clinicians to understand the status of RP and to assess the effect of treatment modalities to the course of the disease. Visual acuity, VF and OCT are among the most commonly applied outcome measures which are used to analyse the progression of the disease. Humphrey VF has been shown to be beneficial in assessing the residual central VF of patients with RP. OCT examination has provided useful information about the pathology and the prognosis of the disease. The studies including OCT have shown that there is a shortening of the EZ length and a thinning of the outer retinal layers in eyes with RP. The EZ is proven to be important for the health and function of the photoreceptors and the integrity of the EZ has been correlated with the severity of RP. Therefore, a combined assessment with OCT and VF is useful for estimating the visual functions of the patients. There are several studies in the literature showing the correlation between retinal structure and vision. The study by Spaide et al. showed that the loss of layers of the outer retina interfered significantly with visual acuity. Pathological studies about RP also confirmed the EZ as the earliest histopathological change in the outer segments of photoreceptors [14] [15].

Colombo et al. analyzed 53 eyes of 27 patients belonging to 12 different family members to assess the annual progression rate of photoreceptor atrophy and EZ line was measured on OCT sections through the fovea. During the 4.84 years mean follow up time, the EZ line width decreased with a yearly average rate of $76.4 \mu \mathrm{m}(4.16 \% /$ year $)$ which was in accordance with the reported rates between $4.9 \%-10.9 \%$ in published literature. [16] [17].

A recent study reviewed medical records of $149 \mathrm{RP}$ patients who reported VF constriction on a central 30-2 Humphrey VF chart. The authors also measured CMT, EZ length, Macular Volume (MV) and Central Choroidal Thickness 
(CCT) with OCT. They reported that BCVA and VF exhibited a progressive worsening related to age and disease duration which were significantly correlated with CMT, EZ length, and MV at the central 1-mm area [18].

Another recent study including 928 patients, evaluated the correlation between MD and visual acuity and found that when MD was lower than $-9.18 \mathrm{~dB}$ the visual acuity would be below $1.0(20 / 20)$. The average decreasing value of $\mathrm{MD}$ in 10 years' period was $-8.01 \pm 3.66 \mathrm{~dB}$ and the value was correlated to retinal pigmentation but not to gender, age or RP family history. They concluded that the value of MD could well reflect the severity of RP [19].

İftikhar et al. established a severity classification system including the parameters of BCVA, VF and OCT. This classification depends on measures that are simple, easy to perform and applicable for almost all patients regardless of any variations in disease phenotype. This classification is also thought to be useful to assess, monitor and compare disease severity in clinical health services and researchs. The authors reported that almost all patients demonstrated a VF extending significantly beyond the edges of their remaining EZ [9]. This led us the opinion that the EZ probably represents organised or densely packed photoreceptors and there may be scattered or fragmented photoreceptors beyond the edges of the EZ that are alive and functioning. The authors suggested that patients who have little to no EZ left could be included to the future clinical trials because they may still have salvageable photoreceptors that may respond to the treatment [9]. In our study, $86 \%$ of the patients were in grade 5 , this means that they had an EZ shorter than 5 degrees. Although the retinal structure was destroyed in most of the patients, we still achieved improvements in BCVA and VF. Our results confirmed the theory of Iftikhar et al. about salvageable photoreceptors.

In recent years stem cell based treatment studies are increasing in number and giving hope for reversing the cell death or damage; or at least causing the functional enhancement of the remaining viable cells. This therapy has been used recently in various ophthalmic diseases which mainly includes hereditary/age related retinal disorders and optic neuropathies and has been shown to have promising results [6] [10] [20] [21] [22] [23] [24]. In order to assess and compare the efficacy of such therapies, it is important to establish outcome measures that are both reliable and easy to evaluate [25]. To date BCVA, VF and Electroretinography (ERG) measurements are used to monitor the treated patients. To the best of our knowledge this is the first clinical study including classification results after stem cell treatment.

In the Reticell-clinical trial, the investigators analyzed the effect of intravitreal use of autologous bone marrow derived MSCs to the quality of life of 20 patients with RP [24]. They assessed the outcome with Visual Function Questionnaire-25 and found a statistically significant improvement in the quality of life of patients 3 months after treatment, whereas by month 12 there was no statistically significant difference from baseline. 
In the largest ophthalmology stem cell clinical trial registered to the "clinicaltrials.gov" 17 patients with bilateral visual loss due to RP were included and followed up at least 6 months. Affected eyes were treated with "The Stem Cell Ophthalmology Treatment Study" (SCOTS) protocol. They evaluated BCVA, VF and OCT to assess the effect of the treatment. In 33 treated eyes, 15 eyes (45\%) improved an average of 7.9 lines of Snellen acuity, 15 eyes (45\%) remained stable, and 3 eyes (10\%) worsened by an average of 1.7 lines of Snellen acuity. The authors did not give detailed information about VF and OCT measurements of the patients [22].

In a recent study, UC-MSC implantation was performed to the suprachoroidal space and no serious ocular adverse effects were reported in this large clinical case series including 124 eyes of $82 \mathrm{RP}$ patients. The authors reported that UC-MSC implantation to the suprachoroidal space demonstrated statistically significant improvements in BCVA, VF and multifocal ERG (mfERG) measurements [10].

In a similar study, Ozmert et al. implanted Wharton's jelly derived MSCs to the subtenon space in $32 \mathrm{RP}$ patients [6]. They reported a significant improvement in the mean BCVA of the patients which increased from 70.5 letters to 80.6 letters at the $6^{\text {th }}$ month. They also found significant improvements in the MD values of VF tests and mean outer retinal thickness measurements, mfERG and full-field flicker ERG evaluations [6].

It is known that stem cell based therapies have better results in patients having more residual visual capacity with greater cellularity and more viable tissue. It is also shown that Retinal Thickness Average (RTA) measurements serve as a diagnostic criterion for stem cell application treatments and better outcomes are revealed in patients with thicker RTA measurements [11] [12]. In a very recent study, Limoli et al. treated 21 eyes of $15 \mathrm{RP}$ patients with autologous adipose tissue derived MSCs into the suprachoroidal space and found that the group with a foveal thickness greater than $190 \mu \mathrm{m}$ is associated with better visual acuity and microperimetric results [11].

To the best of our knowledge this is the first stem cell study in the literature evaluating the effect of stem cell treatment to the severity of RP by using a classification system. The study includes a large sample size and a wide range of patients in terms of age, sex, mode of inheritance and disease duration. Our results showed significant improvements in BCVA and VF after the therapy. The mean score and the grade of the disease also improved after the treatment. We found a negative correlation between VA improvements and scoring and grading of the disease.

We believe that this classification is simple, produces objective measure of disease severity and gives opportunity to compare the results of different treatment modalities.

\section{Limitations}

These are the limitations of our study. 
1) A study of genetics and its correlation with various functional tests were not included in this study. Genetic analysis may guide us to understand the response to the stem cell treatment in different genetic mutations. In an attempt to determine genotypes, we are storing samples from the patients for later genotyping.

2) This study did not include patients with early phase of RP and most of the patients were in grade 5 . Studies comparing different stages of the disease with larger number of patients will help us to identify who will benefit most from stem cell implantations.

3) We believe that electrophysiological tests should be included to the classification. Full field ERG is thought to be the gold standard test for evaluating RP because it is objective and measures global retinal function. Unfortunately, the test is difficult, time-consuming and it requires specialised well-calibrated equipment as well as experienced personnel. Besides by the time most patients are diagnosed it is either undetectable or significantly reduced in amplitude therefore it is impossible to detect meaningful differences. In RP patients, mfERG is a commonly used method for evaluating macular function, so the correlation between the EZ line and mfERG has been studied in RP patients. We believe that including $\mathrm{mfERG}$ as a parameter would increase the value of the classification.

\section{Conclusion}

$\mathrm{RP}$ is a genetic disorder that can cause total blindness with a degeneration process. Currently, gene therapy and cell-based therapies compose the therapeutic options for patients with RP. In order to assess and compare the efficacy of such therapies, it is important to establish outcome measures that are both reliable and easy to evaluate. We believe that this classification is simple, produces objective measure of disease severity and gives opportunity to compare the results of different treatment modalities.

\section{Acknowledgements}

We thank the participants of the study. We would like to thank Prof Dr. Ercument Ovalı and the staff members of Acibadem Labcell for providing the stem cells and Prof Dr Osman Gunay for the statistical analysis. We also thank to the staff members of Acibadem Kayseri Hospital for their contribution to the study tests.

\section{Funding}

This research did not receive any specific grant from funding agencies in the public, commercial or not-for-profit sectors.

\section{Authors' Contributions}

AO: Study design, patient selection and follow-up, surgical intervention, data collection, manuscript preparation 
NS: Patient follow-up, data collection, manuscript preparation

All authors read and approved the manuscript.

\section{Conflicts of Interest}

The authors declare that they have no competing interests.

\section{Ethics Approval and Consent to Participate}

The study was performed in accordance with the Declaration of Helsinki, after obtaining the approval of the Ethics Committee of the University (2017/480, 10.13.2017) and the approval by the Review Board of Stem Cell Applications of the Ministry of Health (Registration number: 56733164/203) according to the regulations in our country. All patients were instructed about the objectives and methodology of the study and gave written informed consent to participate.

\section{References}

[1] Berson, E.L. (1993) Retinitis Pigmentosa. The Friedenwald Lecture. Investigative Ophthalmology \& Visual Science, 34, 1659-1676.

[2] Hartong, D.T., Berson, E.L. and Dryja, T.P. (2006) Retinitis Pigmentosa. The Lancet, 368, 1795-1809. https://doi.org/10.1016/S0140-6736(06)69740-7

[3] Oner, A. (2018) Stem Cell Treatment in Retinal Diseases: Recent Developments. Turkish Journal of Ophthalmology, 48, 33-38. https://doi.org/10.4274/tjo.89972

[4] Zarbin, M. (2016) Cell-Based Therapy for Degenerative Retinal Disease. Trends in Molecular Medicine, 22, 115-134. https://doi.org/10.1016/j.molmed.2015.12.007

[5] Zhang, W., Wang, Y., Kong, J., Dong, M., Duan, H. and Chen, S. (2017) Therapeutic Efficacy of Neural Stem Cells Originating from Umbilical Cord-Derived Mesenchymal Stem Cells in Diabetic Retinopathy. Scientific Reports, 7, Article No. 408. https://doi.org/10.1038/s41598-017-00298-2

[6] Özmert, E. and Arslan, U. (2020) Management of Retinitis Pigmentosa by Wharton's Jelly Derived Mesenchymal Stem Cells: Preliminary Clinical Results. Stem Cell Research \& Therapy, 11, Article No. 25. https://doi.org/10.1186/s13287-020-1549-6

[7] Sujirakul, T., Lin, M.K., Duong, J., Wei, Y., Lopez-Pintado, S. and Tsang, S.H. (2015) Multimodal Imaging of Central Retinal Disease Progression in a 2-Year Mean Follow-up of Retinitis Pigmentosa. American Journal of Ophthalmology, 160, 786-798. https://doi.org/10.1016/j.ajo.2015.06.032

[8] Mitamura, Y., Mitamura-Aizawa, S., Nagasawa, T., Katome, T., Eguchi, H. and Naito, T. (2012) Diagnostic Imaging in Patients with Retinitis Pigmentosa. Journal of Medical Investigation, 59, 1-11.

[9] Iftikhar, M., Lemus, M., Usmani, B., Campochiaro, P.A., Alain Sahel, J., Scholl, H.P.N., et al. (2018) Classification of Disease Severity in Retinitis Pigmentosa. British Journal of Ophthalmology, 103, 1595-1599.

http://dx.doi.org/10.1136/bjophthalmol-2018-313669

[10] Kahraman, N.S. and Oner, A. (2020) Umbilical Cord Derived Mesenchymal Stem Cell Implantation in Patients with Retinitis Pigmentosa: 6-Month Follow-up Results of a Phase 3 Trial. International Journal of Ophthalmology, 13, 1423-1429. https://doi.org/10.18240/ijo.2020.09.14

[11] Limoli, P.G., Limoli, C. and Vingolo, E.M. (2016) Cell Surgery and Growth Factors 
in Dry Age-Related Macular Degeneration: Visual Prognosis and Morphological Study. Oncotarget, 7, 46913-46923. https://doi.org/10.18632/oncotarget.10442

[12] Limoli, P.G., Vingolo, E.M., Limoli, C. and Nebbioso, M. (2019) Stem Cell Surgery and Growth Factors in Retinitis Pigmentosa Patients: Pilot Study after Literature Review. Biomedicines, 7, E94. https://doi.org/10.3390/biomedicines7040094

[13] Kahraman, N.S., Sevim, D.G. and Öner, A. (2019) Cross-Validation of the Turkish Version of the 28-Item Impact of Vision Impairment Profile Test. Open Journal of Ophthalmology, 9, 194-202. https://doi.org/10.4236/ojoph.2019.94021

[14] Spaide, R. and Curcio, C.A. (2011) Anatomical Correlates to the Bands Seen in the Outer Retina by Optical Coherence Tomography. Retina, 31, 1609-1619.

https://doi.org/10.1097/IAE.0b013e3182247535

[15] Sayo, A., Ueno, S., Kominami, T., Okado, S., Inooka, D., Komori, S., et al. (2018) Significant Relationship of Visual field Sensitivity in Central $10^{\circ}$ to Thickness of Retinal Layers in Retinitis Pigmentosa. Investigative Ophthalmology \& Visual Science, 59, 3469-3475. https://doi.org/10.1167/iovs.18-24635

[16] Colombo, L., Montesano, G., Sala, B., Patelli, F., Maltese, P., Abeshi, A., et al. (2018) Comparison of 5-Year Progression of Retinitis Pigmentosa Involving the Posterior Pole among Siblings by Means of SD-OCT: A Retrospective Study. BMC Ophthalmology, 18, Article No. 153. https://doi.org/10.1186/s12886-018-0817-z

[17] Miyata, M., Ogino, K., Gotoh, N., Morooka, S., Hasegawa, T. and Hata, M. (2016) Yoshimura. Inner Segment Ellipsoid Band Length Is a Prognostic Factor in Retinitis Pigmentosa Associated with EYS Mutations: 5-Year Observation of Retinal Structure. Eye, 30, 1588-1592. https://doi.org/10.1038/eye.2016.196

[18] Son, G., Lee, S., Kim, Y.J., Lee, J.Y., Kim, J.G. and Yoon, Y.H. (2019) Correlation between Visual Function and Structural Characteristics of the Macula in Advanced Retinitis Pigmentosa. Ophthalmologica, 242, 22-30. https://doi.org/10.1159/000496941

[19] Ye, H. and Xia, X.P. (2018) Visual Field Mean Deviation and Relevant Factors in 928 Chinese Retinitis Pigmentosa Patients. International Journal of Ophthalmology, 11, 1978-1983. https://doi.org/10.18240/ijo.2018.12.17

[20] Oner, A., Gonen, Z.B., Sevim, D.G., Sinim, N. and Unlu, M. (2018) Suprachoroidal Adipose Tissue Derived Mesenchymal Stem Cell Implantation in Patients with Dry Type Age-Related Macular Degeneration and Stargardt's Macular Dystrophy: 6 Month Follow-up Results of a Phase 2 Study. Cellular Reprogramming, 20, 329-336. https://doi.org/10.1089/cell.2018.0045

[21] Oner, A., Gonen, Z.B., Sevim, D.G., Sinim, N. and Unlu, M. (2019) Six-Month Results of Suprachoroidal Adipose Tissue Derived Mesenchymal Stem Cell Implantation in Patients with Optic Atrophy: A Phase 1/2 Study. International Ophthalmology, 15, 2913-2922. https://doi.org/10.1007/s10792-019-01141-5

[22] Weiss, J.N. and Levy, S. (2018) Stem Cell Ophthalmology Treatmen Study: Bone Marrow Derived Stem Cells in the Treatment of Retinitis Pigmentosa. Stem Cell Investigation, 5, 18. https://doi.org/10.21037/sci.2018.04.02

[23] Weiss, J.N., Levy, S. and Benes, S.C. (2017) Stem Cell Ophthalmology Treatment Study: Bone Marrow Derived Stem Cells in the Treatment of Non-Arteritic Ischemic Optic Neuropathy (NAION). Stem Cell Investigation, 4, 94. https://doi.org/10.21037/sci.2017.11.05

[24] Siqueira, R.C., Messias, A., Messias, K., Arcieri, R.S., Ruiz, M.A., Souza, N.F., Martins, L.C. and Jorge, R. (2015) Quality of Life in Patients with Retinitis Pigmentosa Submitted to Intravitreal Use of Bone Marrow-Derived Stem Cells (Reticell- Clini- 
cal Trial). Stem Cell Research \& Therapy, 14, Article No. 29. https://doi.org/10.1186/s13287-015-0020-6

[25] Jacobson, S.G., Matsui, R., Sumaroka, A. and Cideciyan, A.V. (2016) Retinal Structure Measurements as Inclusion Criteria for Stem Cell-Based Therapies of Retinal Degenerations. Investigative Ophthalmology \& Visual Science, 57, ORSFn1-ORSFn9. https://doi.org/10.1167/iovs.15-17654 